\title{
The Estimation of the Mathematical Exactness of System Dynamics Method on the Base of Some Economic System
}

\author{
Elżbieta Kasperska and Damian Słota \\ Institute of Mathematics, \\ Silesian University of Technology, \\ Kaszubska 23, Gliwice 44-100, Poland, \\ \{elakaspe, damslota\}@polsl.gliwice.pl
}

\begin{abstract}
The purpose of this paper is to present a economic system, such as the base to estimate the mathematical exactness of the System Dynamics Method. An interesting point of view is to examine the reaction of such system to the sinusoidal characteristic of the market sale of a product and to estimate the mathematical preciseness of the method solution.
\end{abstract}

Keywords: System Dynamics Method, Simulation of the System of Production - Store - Sale, Simulation Language Dynamo.

\section{Introduction}

System Dynamics is a well known method of modelling, analysing and simulating dynamic processes occurring in economic and social systems. The world famous theory and application of Forrester [5, 6] and Coyle [1, 2, 3, 4, as well as and many other authors are basically concerned with continuous processes, but an addition of discrete events is also possible [7],8,9]. The attention is paid to the fact that relatively few authors are occupied with estimating the mathematical exactness of the System Dynamics Method. Naturally, this exactness may only be estimated for simple systems (the word "simple" refers to the order of the differential equation which was used to model the system).

In this paper one of such systems is presented. It contains: production, pipeline production, storage and sale. The sinusoidal characteristic of the market demand is assumed. In Figure 1 the scheme of the system assuming Lukaszewicz's symbols [10] is presented with the list of variables and parameters. 


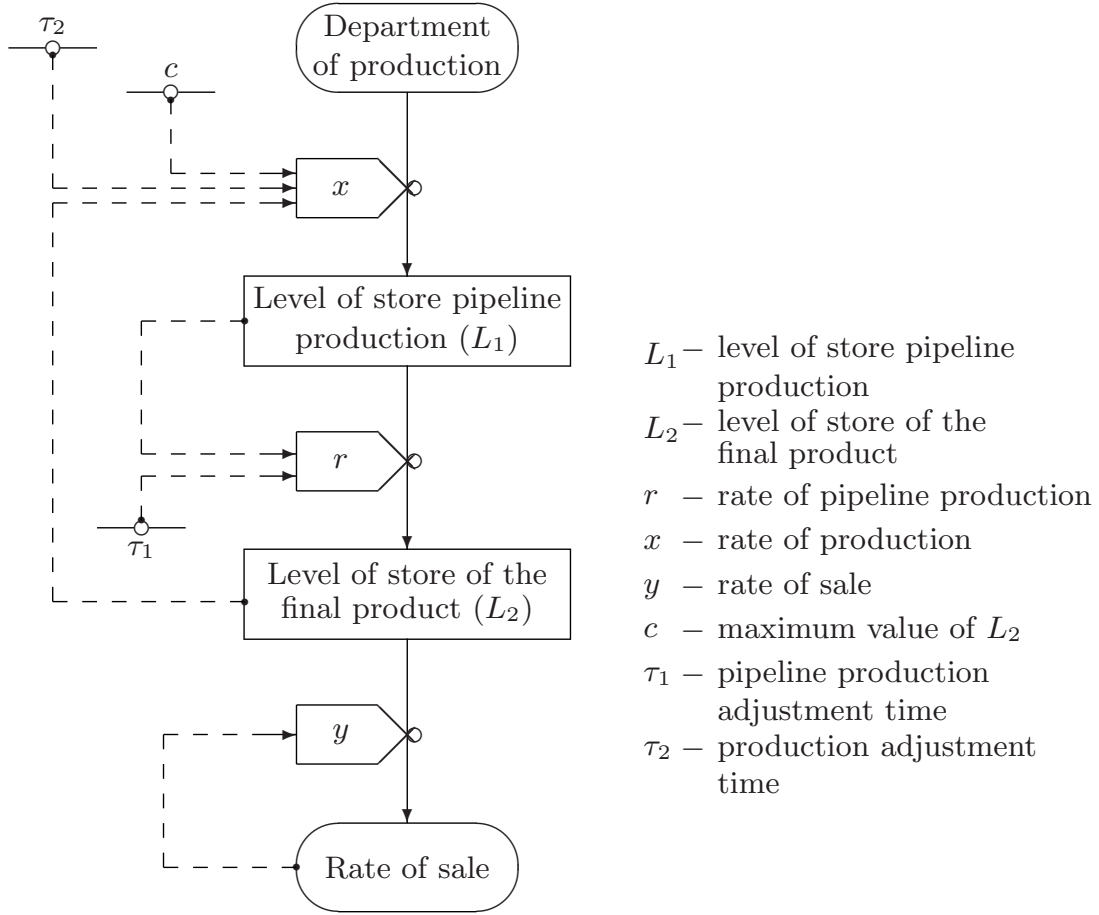

Fig. 1. The production - store - sale system with sinusoidal characteristic of sale

\section{Mathematical Model of the System}

As shown in Figure $\square$ the model consists of five equation:

$$
\begin{aligned}
y(t) & =y(0)+p \sin \left(\frac{2 \pi t}{\omega}\right), \\
x(t) & =\frac{c-L_{2}(t)}{\tau_{2}}, \\
r(t) & =\frac{L_{1}(t)}{\tau_{1}}, \\
\dot{L}_{1}(t) & =x(t)-r(t), \\
\dot{L}_{2}(t) & =r(t)-y(t),
\end{aligned}
$$

with initial conditions:

$$
\begin{aligned}
& x(0)=y(0), \\
& \dot{x}(0)=0 .
\end{aligned}
$$


After prognostic method we obtain (for $\left.\tau_{2}=4 \tau_{1}\right)$ :

$$
\begin{aligned}
x(t)= & \frac{6 \pi \omega^{3} p \tau_{1}+32 \pi^{3} p \omega \tau_{1}^{3}}{\left(\omega^{2}-16 \pi^{2} \tau_{1}^{2}\right)^{2}+64 \pi^{2} \omega^{2} \tau_{1}^{2}} e^{-\frac{t}{2 \tau_{1}}}+ \\
& +\frac{3 \pi \omega^{3} p+16 \pi^{3} p \omega \tau_{1}^{2}-2 \pi p \omega^{3}}{\left(\omega^{2}-16 \pi^{2} \tau_{1}^{2}\right)^{2}+64 \pi^{2} \omega^{2} \tau_{1}^{2}} t e^{-\frac{t}{2 \tau_{1}}}+y(0)+ \\
& +\frac{-6 \pi \omega^{3} p \tau_{1}-32 \pi^{3} p \omega \tau_{1}^{3}}{\left(\omega^{2}-16 \pi^{2} \tau_{1}^{2}\right)^{2}+64 \pi^{2} \omega^{2} \tau_{1}^{2}} \cos \left(\frac{2 \pi t}{\omega}\right)+ \\
& +\frac{p \omega^{4}}{\left(\omega^{2}-16 \pi^{2} \tau_{1}^{2}\right)^{2}+64 \pi^{2} \omega^{2} \tau_{1}^{2}} \sin \left(\frac{2 \pi t}{\omega}\right) .
\end{aligned}
$$

and:

$$
\begin{aligned}
& L_{1}(t)=\left(y(0)+p \sin \left(\frac{2 \pi t}{\omega}\right)\right) \tau_{1}-4 \tau_{1}^{2} \dot{x}(t), \\
& L_{2}(t)=-4 \tau_{1} x(t)+c .
\end{aligned}
$$

So, the total level of storage $L(t)$ is:

$$
L(t)=L_{1}(t)+L_{2}(t)=\left(y(0)+p \sin \left(\frac{2 \pi t}{\omega}\right)\right) \tau_{1}-4 \tau_{1}^{2} \dot{x}(t)-4 \tau_{1} x(t)+c .
$$

In the next section of the paper, we present the results of the simulation of this model usying System Dynamics method and Professional Dynamo 4.0 for Windows.

\section{Simulation of the Dynamics of the Production - Store - Sale System}

The Professional Dynamo 4.0 for Windows provides two types of integration:

- Euler method,

- Runge-Kutta method.

In Table 1 some of the errors in the simulations of these two types are presented. The analysis of errors underlines a considerable exactness, which, in turn, proves the usefulness of the System Dynamics Method, for simulating various economic systems. Moreover, it is used in real chance systems, when their size and complexity make it impossible to apply the exact methods. If the Euler's method is used, attention should be paid to the exactness of the results with a proper choice of the simulation step. Consequently, if the Runge-Kutta method is used, the simulation step is automatically partitioned to obtain a solution for a given exactness (with definite parameter $R E L \_E R R$ ). Moreover, this includes longer calculation time. 
Table 1. The errors in the simulation of the rate of production $x(t)\left(\Delta_{\text {med }}\right.$ - medium absolute error, $\Delta_{\max }$ - maximum absolute error, $\delta_{\text {med }}$ - medium relative error, $\delta_{\max }-$ maximum relative error, $\sigma$ - mean-square error)

\begin{tabular}{||c||c|c|c||c|c|c||}
\hline \hline \multirow{1}{*}{} & \multicolumn{3}{c||}{ Euler method } & \multicolumn{3}{c||}{ Runge-Kutta method } \\
\cline { 2 - 7 } & \multicolumn{3}{|c||}{$d t$} & \multicolumn{3}{c||}{$R E L_{-} E R R$} \\
\cline { 2 - 7 } & 0.1 & 0.5 & 1.0 & 0.1 & 0.01 & 0.001 \\
\hline \hline$\Delta_{\text {med }}$ & 2.62 & 7.49 & 14.35 & 1.99 & 1.99 & 1.98 \\
\hline$\Delta_{\text {max }}$ & 6.85 & 12.89 & 27.60 & 5.67 & 5.67 & 5.65 \\
\hline$\delta_{\text {med }}$ & $7.5110^{-3}$ & $2.8210^{-2}$ & $5.6010^{-2}$ & $4.4410^{-3}$ & $4.4410^{-3}$ & $4.3510^{-3}$ \\
\hline$\delta_{\text {max }}$ & $4.8610^{-2}$ & $2.0510^{-1}$ & $4.1410^{-1}$ & $1.9310^{-2}$ & $1.9310^{-2}$ & $1.8210^{-2}$ \\
\hline$\sigma$ & 3.13 & 8.35 & 15.96 & 2.49 & 2.49 & 2.48 \\
\hline \hline
\end{tabular}

\section{Conclusion}

After modelling and simulation of the production - store - sale system, we have reached the following conclusions:

a) The System Dynamics Method is a very simple universal method, and, at the same time, its mathematical exactness is sufficient or can even be improved with more sophisticated methods of integration (like Runge-Kutta method).

b) The confidence for mathematical exactness of the System Dynamics Method in that paper was consolidated; that fact can help for development both:

- further work for sophisticated tools of simulation complex system,

- further application of the System Dynamics Method.

\section{References}

1. Coyle, R. G.: Management System Dynamics. John Wiley \& Sons, New York (1977)

2. Coyle, R. G., Wolsterholm, E. P.: Modelling discret events in System Dynamics model. A case study. Dynamica 6 (1980) 21-27

3. Coyle, R. G.: System Dynamics Modelling. A Practical Approach. Chapman \& Hall, London (1996)

4. Coyle, R. G.: The practice of System Dynamics: milestones, lessons and ideas from 30 years experience. System Dynamics Rev. 14 (1998) 343-365

5. Forrester, J. W.: Industrial Dynamics. MIT Press, Massachusetts (1961)

6. Forrester, J. W.: Principles of Systems. Cambridge Press, Massachusetts (1972)

7. Kasperska, E., Mateja-Losa, E., Słota, D.: Some extension of System Dynamics method - practical aspects. In Deville, M., Owens, R. (eds.): Proc. 16th IMACS World Congress. IMACS, Lausanne (2000) 718-11 1-6

8. Kasperska, E., Słota, D.: Two different methods of embedding the optimization in simulation on model DYNBALANCE(2-2). In Davidsen, P. I., Mollona, E. (eds.): Proc. 20th Int. Conf. of the System Dynamics Society. SDS, New York (2003) 1-23

9. Kasperska, E., Słota, D.: Mathematical Method in the Management in Conceiving of System Dynamics. Silesian Technical University, Gliwice (2000) (in Polish)

10. Łukaszewicz, R.: Management System Dynamics. PWN, Warsaw (1975) (in Polish). 